\title{
Management of a Unique Sinonasal Undifferentiated Carcinoma Subtype in the Era of SARS-CoV-2
}

\author{
Jennifer E. Douglas ${ }^{a} \quad$ Adam C. Kaufman $^{a} \quad K^{2}$ arthik Rajasekaran ${ }^{a}$ \\ a Department of Otorhinolaryngology - Head and Neck Surgery, University of Pennsylvania \\ Health System, Philadelphia, PA, USA
}

\section{Keywords}

Sinonasal undifferentiated carcinoma - Sinonasal cancer - SMARCB-deficient carcinoma .

Skull base surgery $\cdot$ COVID-19

\begin{abstract}
The novel coronavirus (SARS-CoV-2) pandemic has influenced the timeliness of care for patients with both common and rare conditions, particularly those affecting high-risk operative sites such as the upper aerodigestive tract. Sinonasal undifferentiated carcinoma (SNUC) represents a rare malignancy of the sinonasal tract, a unique subset of which has never been previously reported in the otolaryngology literature and is characterized by inactivation of the SMARCB (INI-1) tumor suppressor gene. This subtype exhibits a particularly poor prognosis and is characterized pathologically by its rhabdoid appearance. Here we present the case of an individual who was diagnosed with a sinonasal mass during the SARS-CoV-2 pandemic, which was ultimately found to be SMARCB (INI-1)-deficient sinonasal carcinoma. Advanced imaging was deferred in the interest of limiting the patient's exposure to the virus, and expedited operative management was performed which facilitated prompt referral for adjuvant chemoradiation. The SARS-CoV-2 pandemic presents unique challenges, but the work-up of high-risk lesions must be prioritized; this continues to be paramount as SARS-CoV-2 resurges in many cities across the USA.

(C) 2020 S. Karger AG, Basel
\end{abstract}

This research was selected for presentation at the 2020 meeting of the American Rhinologic Society at the American Academy of Otolaryngology - Head and Neck Surgery. 


\begin{tabular}{l|l}
\hline ORL 2021;83:47-51 \\
\hline DOI: 10.1159/000511713 & $\begin{array}{l}\text { @ 2020 S. Karger AG, Basel } \\
\text { www.karger.com/orl }\end{array}$ \\
\hline
\end{tabular}

Douglas et al.: Management of SNUC during COVID-19

\section{Introduction}

Sinonasal undifferentiated carcinoma (SNUC) is a rare poorly differentiated malignancy arising from the sinonasal tract. While SNUC has itself a poor prognosis, a subset of sinonasal neoplasms with inactivation of the SMARCB (INI-1) tumor suppressor gene exhibits particularly treatment-recalcitrant disease. This was first reported in the pathology literature in 2014 [1]; however, it has yet to be detailed in the otolaryngology literature. Here we present the case of an individual with a sinonasal mass who was ultimately diagnosed with SMARCB (INI-1)-deficient sinonasal carcinoma and the unique management required during the SARSCoV-2 pandemic.

SWI/SNF-related matrix-associated actin-dependent regulator of chromatin subfamily B member 1, also known as SMARCB1or INI-1, is a transcription regulator located on chromosome 22q11.2 that functions as a tumor suppressor gene [2]. It is ubiquitously expressed in normal tissue and it has been implicated in the pathogenesis of a number of "rhabdoid"appearing malignancies including renal medullary carcinoma, myoepithelial carcinoma, epithelioid carcinoma, and epithelioid malignant nerve sheath tumors [3-5]. It was first identified in sinonasal malignancies in 2014, when Bishop et al. [1] found that nearly $6 \%$ of primary sinonasal carcinomas exhibited inactivation of SMARCB1. These cases were initially classified as nonkeratinizing squamous cell carcinomas $(n=3)$, sinonasal undifferentiated carcinomas $(n=2)$, myoepithelial carcinomas $(n=2)$, nonintestinal adenocarcinomas $(n=1)$, and carcinoma, not otherwise specified $(n=1)$ [1] and presented at advanced stages with a propensity for local recurrence and metastatic disease, increasing the overall patient mortality [6].

Despite knowledge of the mutation underlying SMARCB (INI-1)-deficient sinonasal carcinoma, its existence has not been discussed in the otolaryngology literature. Here we present the case of an individual initially diagnosed with nasal polyps who was ultimately diagnosed with SMARCB (INI-1)-deficient sinonasal carcinoma during the SARS-CoV-2 pandemic and the unique management required.

\section{Case Report}

A 60-year-old female presented for evaluation of nasal congestion refractory to conservative measures. Nasal endoscopy identified a left nasal mass initially felt to represent nasal polyps; however, due to the extensive nature of the disease, the patient was referred to our institution for a second opinion. A noncontrast-enhanced computed tomography (CT) scan of the sinuses was performed, which demonstrated a $5.0 \times 1.6 \times 4.0 \mathrm{~cm}$ left nasal cavity mass with extensive osseous remodeling (Fig. 1), suspicious for malignancy. Ordinarily, an in-office biopsy would have been performed and a magnetic resonance imaging (MRI) scan would have been ordered to better characterize the extent of the disease. However, due to the SARS-CoV-2 pandemic, we elected against both to mitigate the risk of: (1) additional viral exposure for the patient due to hospital visits for MRI scans and (2) exposure of office staff to aerosolizing procedures.

The patient was counseled on the concerning findings and the current health care climate. The options were to go to the operating room for a biopsy alone versus biopsy and resection if the pathology was consistent with a malignancy. The patient elected the latter option. The patient was thus taken to the operating room by the otolaryngology team, and a biopsy confirmed malignancy on a frozen specimen. A more specific diagnosis was unable to be made and, per the preoperative discussion, a comprehensive endoscopic resection was subsequently performed. Due to tumor extending to the cribriform plate, a small cerebrospinal fluid leak was encountered which was repaired with a nasoseptal flap and an abdominal fat graft. The patient was observed in the postanesthesia care unit and discharged home without issue that evening. The final pathology showed SMARCB1 (INI-1)-deficient sinonasal carcinoma (Fig. 2). Endoscopic examination 3 and 6 weeks postoperatively showed no evidence of persistent cerebrospinal fluid leak. Margins were clear but, given the pathology, the patient was referred for adjuvant chemoradiation therapy and is currently midway through treatment. 


\begin{tabular}{ll}
\cline { 2 - 2 } ORL 2021;83:47-51 \\
\cline { 2 - 3 } DOI: 10.1159/000511713 & $\begin{array}{l}\text { (c) 2020 S. Karger AG, Basel } \\
\text { www.karger.com/orl }\end{array}$ \\
\cline { 2 - 3 }
\end{tabular}

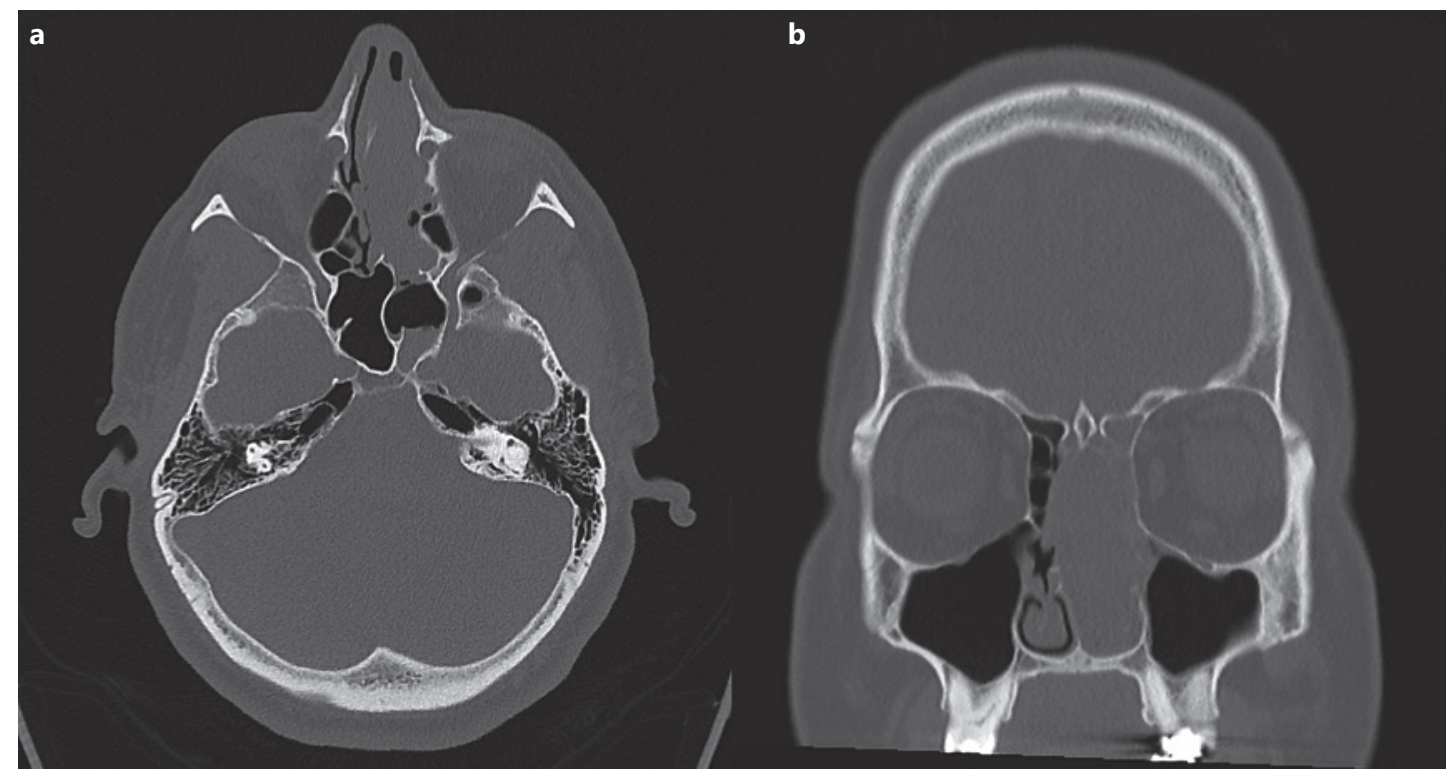

Fig. 1. Pre-operative axial (a) and coronal (b) views of a non-contrast-enhanced CT scan of the sinuses showing a 5.0-cm mass of the left nasal cavity involving the turbinates and the ethmoid sinus with osseous remodeling.

Fig. 2. Pathology of the specimen confirmed to be SMARCB1 (INI-1)-deficient sinonasal carcinoma, which is characterized by a rhabdoid appearance with large cells with abundant eosinophilic cytoplasm and eccentrically placed nuclei with open chromatin and prominent nucleoli.

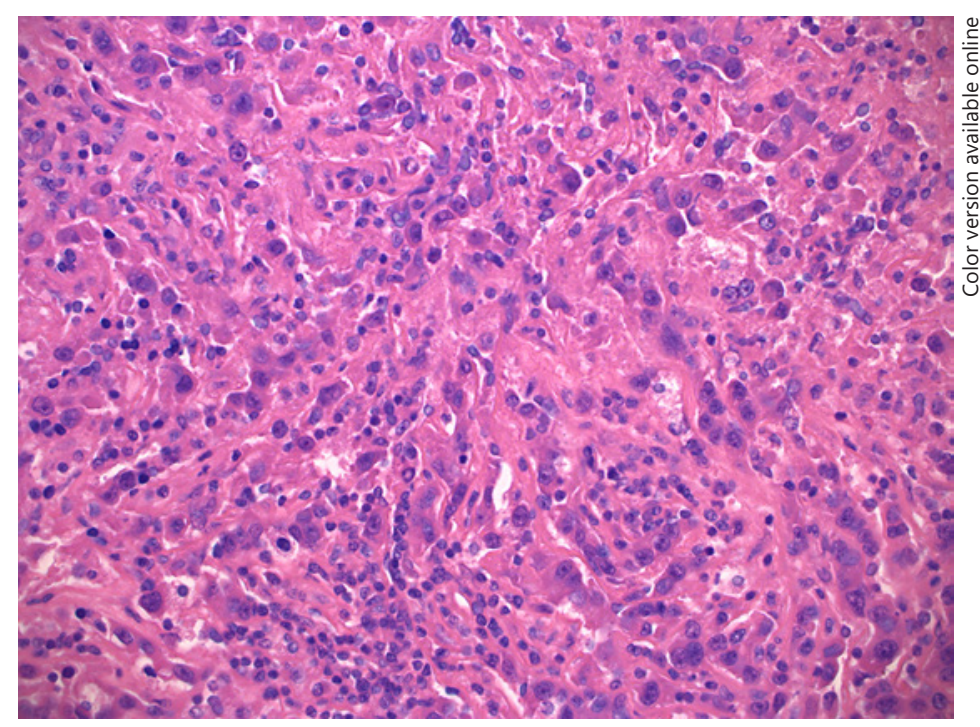

\section{Discussion}

The SARS-CoV-2 pandemic has limited inpatient, outpatient, and surgical volumes, as well as access to hospital resources including ancillary services like radiology and speech language pathology. Otolaryngology patients represent a particularly high-risk population given the anatomic site of disease often involving nasopharyngeal or oropharyngeal mucosa. This has been particularly recognized within the subspecialty of rhinology and skull base surgery [7]. Given this, it was deemed best to defer an MRI scan preoperatively for this patient to limit her exposure to the healthcare system and to expedite her surgical procedure. Irre- 
Douglas et al.: Management of SNUC during COVID-19

spectively of the MRI result, the patient warranted a biopsy of the lesion due to its concerning appearance. Moreover, performing intraoperative frozen sectioning and then proceeding immediately to resection without delay while awaiting the definitive pathology results further limited healthcare exposure. The patient thus underwent a streamlined course of treatment from presentation through diagnosis, treatment, and referral to the appropriate consultants.

In the era of the SARS-CoV-2 pandemic, otolaryngology patients represent a unique subset of patients who must be managed with particular care given the high-risk anatomic sites involved and the frequency of aerosol-generating procedures. When working up an atypical sinonasal mass, it is important to consider the rare case of SNUC variant SMARCB (INI-1)-deficient sinonasal carcinoma as a potential diagnosis. The work-up and treatment of this pathology must be expedited under even ideal circumstances, and its management during the SARS-CoV-2 pandemic is challenging from both a patient and a physician standpoint. Nevertheless, it warrants a prioritized work-up and thorough patient counseling given the propensity for late-stage presentation and rapid progression. This is particularly relevant with the recent resurgence of SARS-CoV-2. Further studies are necessary to better characterize the particular clinical characteristics of this SNUC variant to define clear diagnostic and treatment measures.

\section{Acknowledgment}

The authors would like to acknowledge the contributions of Dr. Ming Zhang, MD, PhD, Assistant Professor of Pathology and Laboratory Medicine at Pennsylvania Hospital, for his assistance in review of and insight into this unique surgical pathology.

\section{Statement of Ethics}

This research complies with the guidelines for human studies and was conducted ethically in accordance with the World Medical Association Declaration of Helsinki. The subject gave her written informed consent for publication of this case (including publication of the images). This case report was also reviewed by the University of Pennsylvania Institutional Review Board and was deemed exempt from the requirement of full board review.

\section{Conflict of Interest Statement}

The authors declare no relevant conflict of interests.

\section{Funding Sources}

No financial support was received for this project.

\section{Author Contributions}

J.E.D.: literature review and drafting and revision of this paper. A.C.K.: literature review and revisions of this paper. K.R.: project conceptualization and revision of this paper. 


\section{References}

1 Bishop JA, Antonescu CR, Westra WH. SMARCB1 (INI-1)-deficient carcinomas of the sinonasal tract. Am J Surg Pathol. 2014 Sep;38(9):1282-9.

2 Hollmann TJ, Hornick JL. INI1-deficient tumors: diagnostic features and molecular genetics. Am J Surg Pathol. 2011 Oct;35(10):e47-63.

3 Hornick JL, Dal Cin P, Fletcher CD. Loss of INI1 expression is characteristic of both conventional and proximaltype epithelioid sarcoma. Am J Surg Pathol. 2009 Apr;33(4):542-50.

4 Gleason BC, Fletcher CD. Myoepithelial carcinoma of soft tissue in children: an aggressive neoplasm analyzed in a series of 29 cases. Am J Surg Pathol. 2007 Dec;31(12):1813-24.

5 Cheng JX, Tretiakova M, Gong C, Mandal S, Krausz T, Taxy JB. Renal medullary carcinoma: rhabdoid features and the absence of INI1 expression as markers of aggressive behavior. Mod Pathol. 2008 Jun;21(6):647-52.

6 Parsel SM, Jawad BA, McCoul ED. SMARCB1-Deficient Sinonasal Carcinoma: Systematic Review and Case Report. World Neurosurg. 2020 Apr;136:305-10.

7 Patel ZM, Fernandez-Miranda J, Hwang PH, Nayak JV, Dodd R, Sajjadi H, et al. Letter: Precautions for Endoscopic Transnasal Skull Base Surgery During the COVID-19 Pandemic. Neurosurgery. 2020 Jul;87(1):E66-7. 